\section{Gradiente urbano-rural no padrão de consumo e cessação do tabagismo no Brasil}

\author{
Urban-rural gradient in tobacco consumption and \\ cessation patterns in Brazil
}

\author{
Gradiente urbano-rural en el patrón de consumo \\ y fin del tabaquismo en Brasil
}

\section{Resumo}

O grau de urbanização tem sido associado ao consumo de produtos do tabaco, bem como a interrupção do hábito de fumar. Dito isso, o objetivo deste trabalho é examinar a importância do contexto urbano-rural na determinação do padrão de consumo de derivados do tabaco e da cessação do hábito de fumar. Utilizou-se dados do módulo suplementar Pesquisa Especial de Tabagismo (PETab) do inquérito Pesquisa Nacional por Amostra de Domicílios (PNAD) de 2008. Foram analisados 14.420 indivíduos maiores de 15 anos, sendo 7.003 tabagistas atuais e 7.417 ex-tabagistas na PETab. Foram calculados a prevalência e o indice de cessação de tabagismo. Houve uma estratificação espacial desses indivíduos por moradores de ambientes urbanos e rurais. Outras variáveis sociodemográficas também foram analisadas: sexo, faixa etária, raça e escolaridade. Para explorar a diferença na prevalência e na cessação de tabagismo para cada estrato da situação censitária, os dados foram ajustados usando-se um modelo de Poisson. Há uma prevalência de tabagismo maior para os locais menos influenciados pelo ambiente urbano. Por outro lado, a cessação do tabagismo é maior nos locais mais urbanizados. $\mathrm{Ob}$ serva-se que para a prevalência de tabagismo há redução no consumo na medida em que aumenta a característica urbana da população $(R P=0,75$ para eminentemente urbana vs. eminentemente rural, $p=0,001)$. Para a cessação do tabagismo, observa-se que a população mais urbana é a mais propensa à interrupção do consumo $(R P=1,22$ para eminentemente urbana vs. eminentemente rural, $p<0,001)$. Os resultados apontam para a necessidade de aperfeiçoar a efetividade dos serviços de acompanhamento e estímulo à cessação para o ambiente rural.

Fumar; Abandono do Hábito de Fumar; Tabagismo; População Urbana; População Rural

\author{
Camila Drumond Muzi 1,2 \\ Valeska Carvalho Figueiredo 3 \\ Ronir Raggio Luiz 2
}

doi: 10.1590/0102-311X00077617

\author{
Correspondência \\ C. D. Muzi \\ Instituto Nacional de Câncer José Alencar Gomes da Silva. \\ Praça da Cruz Vermelha 23, Rio de Janeiro, RJ 20230-130, \\ Brasil. \\ camilamuzi@gmail.com \\ 1 Instituto Nacional de Câncer José Alencar Gomes da Silva, Rio \\ de Janeiro, Brasil. \\ 2 Instituto de Estudos em Saúde Coletiva, Universidade Federal \\ do Rio de Janeiro, Rio de Janeiro, Brasil. \\ 3 Escola Nacional de Saúde Pública Sergio Arouca, Fundação \\ Oswaldo Cruz, Rio de Janeiro, Brasil.
}




\section{Introdução}

A mortalidade anual mundial relacionada ao tabaco é de 5,4 milhões de pessoas e, em torno de $70 \%$ destes óbitos ocorrem em países em desenvolvimento. $\mathrm{O}$ tabagismo como fator de risco para as doenças não transmissíveis só é ultrapassado pela a hipertensão arterial sistêmica 1.

Desde o final da década de 1980, o Brasil vem promovendo ações de redução do tabagismo, obtendo sucesso reconhecido internacionalmente no seu controle principalmente por meio do aumento da tributação e o estímulo ao uso de propagandas antitabaco 2 . Como resultado, a prevalência de tabagismo reduziu de 32,4\% em 1989, para 17\% em 2008, e 14,7\% em 20133.

De uma forma geral, a redução da prevalência de tabagismo tem duas abordagens principais: a prevenção da iniciação e o incentivo à cessação tabágica ${ }^{4}$. As estratégias adotadas nas políticas mudam de acordo com a sua efetividade e com o contexto em que elas ocorrem ${ }^{5}$. Tendo em vista que a cessação do fumo um desafio atual em todo o mundo 1 , é importante reconhecer que inúmeros fatores são determinantes para tal fim, entre os quais se incluem fatores proximais, tais como sexo, faixa etária, condição socioeconômica e, sobretudo, aqueles distais ou contextuais, como a urbanização, por exemplo 6 .

Em particular, o grau de urbanização tem sido associado ao tabagismo em estudos mais recentes, frequentemente relacionado a um marketing agressivo e a ambientes regulatórios fracos 7 . Nesse sentido, o baixo grau de urbanização, representado pelas populações rurais, apresenta historicamente associação com o consumo de produtos do tabaco por outras razões como condição socioeconômica inferior e dependência financeira, quando se trata de área de produção fumageira. Ademais, comunidades rurais tendem a ter um acesso maior a cigarros contrabandeados (ilícitos) e a sofrer menor acesso e impacto de informações de conscientização dos efeitos do tabagismo pois possuem, em geral, menor escolaridade 8 .

Considera-se, portanto, que as estratégias de mercado para o estímulo ao consumo de tabaco podem ter impactos diferentes de acordo com o grau de urbanização. Além disso, como o alcance das estratégias de cessação de tabagismo também podem se comportar de forma diferenciada em ambientes mais urbanizados, é importante estimar a diferença destas duas características nos diversos contextos socioespaciais 6,9. A hipótese que norteia este estudo, portanto, é a de que os ambientes urbano e rural apresentam padrões diferentes de tabagismo e de cessação, e que há uma mudança gradativa nestes dois indicadores na medida em que muda o perfil de urbanização das localidades. Nesse sentido, o objetivo deste trabalho é investigar a presença de um gradiente urbano-rural na prevalência e cessação de tabagismo.

\section{Metodologia}

Este é um estudo observacional do tipo transversal que utilizou dados do módulo suplementar Pesquisa Especial de Tabagismo (PETab) da Pesquisa Nacional por Amostra de Domicílios (PNAD) de 200810. A PETab é uma pesquisa adaptada do Global Adult Tobacco Survey (GATS) que foi realizado em 22 países do mundo com o apoio técnico da Organização Mundial da Saúde (OMS) e do Center for Disease Control and Prevention (CDC), além do financiamento da Filantropia Bloomberg para que se investigasse a prevalência e as características do tabagismo no mundo por meio de um método padronizado. O estudo faz parte do Sistema Global de Vigilância do Tabagismo (Global Tobacco Surveillance System) 11.

A PETab consistiu num plano amostral semelhante ao da PNAD 2008, que correspondeu a cerca de $1 / 3$ das unidades de habitação selecionadas para ela. Essa pesquisa nacional consistiu em uma amostra probabilística complexa com 3 estágios de seleção, e a PETab contou com um quarto estágio adicional que foi a seleção de um morador com 15 anos ou mais de idade (sem admitir a coleta de informações por proxy, como foi permitido na PNAD) ${ }^{10}$. Maiores detalhes metodológicos estão nas publicações de referência do Instituto Brasileiro de Geografia e Estatística (IBGE) 10. 


\section{Amostra de estudo}

Foram consideradas para o presente trabalho as 39.425 pessoas participantes da PETab, das quais: 14.420 indivíduos maiores de 15 anos, sendo 7.003 tabagistas atuais; 7.417 ex-tabagistas; e 25.005 que nunca fumaram. Com base nessas informações foram estimadas a prevalência de tabagismo e o índice de cessação de tabagismo, que é a proporção de pessoas que parou de fumar dentre todos os tabagistas e ex-tabagistas no momento da pesquisa.

\section{Instrumentos e coleta de dados}

O questionário da PETab de 2008 conta com questões específicas para as seguintes condições de uso do tabaco: nunca foram usuários de tabaco; são usuários atuais de produto do tabaco fumado; e não são fumantes atuais, mas são ex-usuários. A essas pessoas foram dirigidas questões detalhadas, principalmente, sobre os seguintes aspectos: características do tabagismo, incluindo o tipo de produto do tabaco utilizado; idade em que começou a fumar; e tentativas de cessação. Complementarmente, abordou-se a exposição passiva à fumaça do tabaco e a mídia, tanto como veículo de propaganda de cigarros quanto de campanhas de informações sobre os riscos do fumo 10 .

O status de tabagismo foi definido baseando-se em duas perguntas: "Atualmente o(a) Sr(a) fuma algum produto do tabaco?". Foram considerados fumantes aqueles que responderam fumar "diariamente" ou "menos que diariamente". Aqueles que responderam não serem fumantes no momento da pesquisa, arguiu-se: "E no passado, o(a) Sr(a) fumou algum produto do tabaco?". Foram considerados ex-fumantes os indivíduos que responderam ter fumado diariamente ou menos que diariamente no passado. A soma de fumantes e ex-fumantes caracteriza o grupo de indivíduos que foram fumantes em algum período da vida.

\section{Variáveis do estudo}

\section{a) Variável de interesse}

Houve uma estratificação espacial desses indivíduos por moradores de ambientes urbanos e rurais. Para tal, foi utilizada a variável "situação censitária”, que originalmente é dividia em oito estratos 12: "URBANA - cidade ou vila, área urbanizada”; "URBANA - cidade ou vila, área não urbanizada”; "URBANA - área urbana isolada"; "RURAL - aglomerado rural de extensão urbana"; "RURAL - aglomerado rural, isolado, povoado"; "RURAL - aglomerado rural, isolado, núcleo"; "RURAL - aglomerado rural, isolado, outros aglomerados"; "RURAL - zona rural exclusive aglomerado rural”. A variável foi recodificada em 4 categorias: "eminentemente urbano", "urbano periférico", "rural com influência urbana", "eminentemente rural".

\section{b) Variável resposta}

Foram calculados a prevalência e o índice de cessação de tabagismo. A prevalência trata da razão entre o número de fumantes atuais de tabaco mais fumantes ocasionais sobre todos os indivíduos da amostra. Por outro lado, a cessação infere a razão entre ex-fumantes (numerador) e o total de fumantes em algum período da vida, em que o denominador é a soma de ex-fumantes e fumantes atuais.

\section{c) Covariáveis}

Outras variáveis sociodemográficas também foram analisadas: sexo (masculino e feminino), faixa etária ( $\leq 24$ anos, 25-39 anos, 40-59 e $\geq 60$ anos), raça (brancos e não brancos), uso de serviços de saúde nos últimos 12 meses, doenças crônicas relacionadas ao tabaco (incluindo câncer em geral, doenças pulmonares obstrutivas crônicas como bronquite e asma e, por fim, doenças do coração), escolaridade (analfabeto e Ensino Fundamental incompleto; Ensino Fundamental completo e Ensino Médio incompleto; e Ensino Médio completo e mais). 


\section{Análise estatística}

A prevalência de tabagismo e o índice de cessação de tabagismo foram estimados com base na frequência do status de tabagismo, respectivamente, entre o conjunto de participantes da PETab e o conjunto de fumantes (ex-usuários e atuais). Para comparar a frequência em cada categoria das covariáveis foi utilizado o $\chi^{2}$ de Pearson e seu respectivo valor de $\mathrm{p}$. De forma análoga, a fim de observar a presença de gradiente nas prevalências de tabagismo e cessação de tabagismo (por meio do índice de cessação de tabagismo) entre as categorias ordinais de mais de duas categorias, foi calculado o $\chi^{2}$ de tendência com o respectivo valor de p.

Para explorar a diferença na prevalência e na cessação de tabagismo para cada estrato da situação censitária, os dados foram ajustados por meio de um modelo de Poisson. Foram incluídas no modelo as variáveis demográficas analisadas, cuja análise bivariada indicou associação estatisticamente significativa com os desfechos estudados. As variáveis explicativas foram testadas individualmente e acrescentadas no modelo sempre que apresentassem significância estatística $(\mathrm{p}<0,05)$ usando-se o método Forward. Foram ainda testados termos de interação no modelo. As categorias adotadas como referência para essas variáveis foram escolhidas considerando-se sempre aquela com a menor prevalência. Na modelagem, para cada variável foram calculadas as razões de prevalência bruta e ajustada, de acordo com o melhor modelo obtido pelas estatísticas de máxima verossimilhança. Foram ainda estimados os intervalos de confiança a um nível de 95\% de significância.

Cabe ressaltar que todas as análises foram realizadas mediante a incorporação do esquema amostral complexo da PNAD 2008. Por fim, obedecendo à Resolução no 466/2012, dispensa-se a aprovação de Comitê de Ética em Pesquisa (CEP) por se tratar de dados públicos e sem qualquer identificação dos sujeitos entrevistados.

\section{Resultados}

A Tabela 1 apresenta a prevalência e a cessação do tabagismo para cada situação censitária. Há uma prevalência de tabagismo maior para os locais menos influenciados pelo ambiente urbano. Por outro lado, a cessação do tabagismo é maior nos locais mais urbanizados. Vale destacar que há uma formação de gradiente entre as categorias ordinais da variável situação censitária, tanto para a prevalência quanto para a cessação do tabagismo (respectivamente, $\mathrm{p}<0,001$ e p =0,007).

Para a análise da estimativa de associação entre as variáveis preditoras e a prevalência e cessação de tabagismo, foi realizada a modelagem usando-se a regressão de Poisson, e por meio dela foram calculadas as respectivas razões de prevalência. Os resultados estão apresentados, respectivamente, nas Tabelas 2 e 3.

Valendo-se das razões de prevalência (RP) e seus respectivos intervalos de $95 \%$ de confiança (IC95\%), observa-se que para a prevalência de tabagismo há uma redução do consumo na proporção em que aumenta a característica urbana da população: RP = 0,98 (IC95\%: 0,84-1,16) para rural com influência urbana; $R P=$ 0,91 (IC95\%: 0,76-1,09) para urbana periférica; e RP = 0,74 (IC95\%: 0,70-0,79) para eminentemente urbana $v s$. eminentemente rural. A medida é estatisticamente significativa para a população eminentemente urbana $(\mathrm{p}=0,001)$, havendo ainda um gradiente nos estratos, reduzindo a prevalência de tabagismo na medida em que aumenta a urbanidade ( $\mathrm{p}$ de tendência $=0,042$ ). Ainda, observa-se que o grupo de tabagistas corresponde a uma população masculina, mais velha, de não brancos e com menor escolaridade, sem referir uso de serviços de saúde nos últimos 12 meses, e menor prevalência de doenças crônicas relacionadas ao tabaco (Tabela 2).

Para a cessação do tabagismo, observa-se que a população mais urbana é a mais propensa à interrupção do consumo: $\mathrm{RP}=1,13$ (IC95\%: 1,06-1,20) para rural com influência urbana; $\mathrm{RP}=1,21$ (IC95\%: 1,04-1,40) para urbana periférica; e RP = 1,23 (IC95\%: 1,07-1,39) para eminentemente urbana vs. eminentemente rural, sendo todos os estratos significativos (respectivamente, $\mathrm{p}=0,013 ; \mathrm{p}=0,006$ e p $<0,001$ ), com gradiente entre as categorias ( $\mathrm{p}$ de tendência =0,021). Observa-se, ainda, que há uma prevalência maior de ex-fumantes entre o sexo feminino, em idades mais avançadas, brancos, com maior escolaridade, que referiram uso de serviços de saúde nos últimos 12 meses, e com maior prevalência de doenças crônicas relacionadas ao tabaco (Tabela 3). 
Tabela 1

Prevalência de tabagismo e índice de cessação do tabagismo segundo situação censitária pela Pesquisa Nacional por Amostra de Domicílios. Brasil, 2008.

\begin{tabular}{|c|c|c|c|c|}
\hline Situação censitária & Prevalência de tabagismo & $\begin{array}{l}\text { Valor de p de } \\
\text { tendência }\end{array}$ & $\begin{array}{c}\text { Índice de cessação de } \\
\text { tabagismo }\end{array}$ & Valor de $p$ de tendência \\
\hline Eminentemente urbana & 16,9 & $<0,001$ & 52,3 & 0,007 \\
\hline Urbana periférica & 18,4 & & 50,0 & \\
\hline $\begin{array}{l}\text { Rural com influência } \\
\text { urbana }\end{array}$ & 19,1 & & 47,0 & \\
\hline Eminentemente rural & 22,8 & & 46,5 & \\
\hline Total & 17,76 & & 51,44 & \\
\hline
\end{tabular}

\section{Discussão}

O fenômeno da urbanização é uma questão mundial, bem como as suas consequências na saúde dos indivíduos 13. A maior prevalência de tabagistas no ambiente rural não é recente no Brasil e é avaliada há pelo menos 15 anos. Em 1989, o Inquérito Nacional de Saúde e Nutrição, realizado em adultos acima de 18 anos, estimou a prevalência de tabagismo em homens por tipo de ambiente: 41,1\% urbano vs. 50\% rural, e nas mulheres: 26,5\% urbano vs. 29\% rural. Já em 2003, a Pesquisa Mundial de Saúde estimou decréscimos em todas as prevalências de tabagismo vigentes: em homens 26,2\% nas áreas urbanas $v s$. $31,8 \%$ na área rural, e nas mulheres $18,2 \%$ urbano vs. $19,6 \%$ rural ${ }^{3}$.

Estudos internacionais também se preocuparam em avaliar as diferenças entre ambientes urbanos e rurais e seus impactos na tendência de tabagismo. Entre 1985-2000, Idris et al. 14 avaliaram os padrões de tabagismo em 279.278 indivíduos entre 25-79 anos em 6 países europeus: Finlândia, Suécia, Dinamarca, Alemanha, Itália e Espanha. Foi encontrada, de forma geral, uma prevalência maior em áreas urbanas com alto grau de urbanização, e presença de maior desigualdade entre mulheres em comparação aos homens e nos indivíduos de baixa escolaridade em comparação aos de alta escolaridade. Finlândia e Espanha apresentaram altas taxas de tabagismo independentemente de o ambiente ser urbano ou rural, 46,6 e 46,3\% entre finlandeses e 49,6 e 50,5\% nos espanhóis. Na China, inquéritos nacionais estimaram a prevalência de fumantes em 1993 (32,2\%), 1998 (28,9\%) e em 2003 (26\%). As taxas de cessação foram mais altas em áreas urbanas do que em rurais (17,3\% em área urbana vs. 15,3\% em área rural) 15. Dados de um inquérito de saúde norte-americano de 2007 apontam que a prevalência nacional de tabagismo em adultos que vivem em áreas não metropolitanas é de 24,5\%, pequenas áreas metropolitanas $(20,9 \%)$ e grandes áreas metropolitanas $(17,4 \%)$ 16. Outro estudo que traçou o perfil de tabagismo em áreas rurais no mesmo país, identificou a alta prevalência de adultos fumantes em áreas de cultura de tabaco como o Estado de Kentucky, Estados Unidos, com 31,8\% 17. Cabe ressaltar que a associação entre prevalência de tabagismo e residir em área rural foi ainda observada em alguns países que realizaram o GATS, tais como Bangladesh 18, China 19 e Índia 20.

Tejada et al. 21 estimaram os determinantes da cessação de tabagismo, bem como as características sociodemográficas dos indivíduos entrevistados na PETab. A prevalência de cessação no ambiente urbano foi maior do que no rural (48,80 vs. 45,25\%), com diferença estatisticamente significativa (valor de $\mathrm{p}=0,019$ ). Os autores estimaram que a RP bruta foi $8 \%$ maior no ambiente urbano com significância estatística (valor de $\mathrm{p}=0,022$ ). Porém, ao realizar a modelagem multivariada quando ajustada por demais variáveis sociodemográficas e de saúde, houve perda da significância estatística e do poder de associação ( $R P=1,01$; IC95\%: 0,94-1.08), sugerindo que estes seriam fatores importantes na determinação do perfil urbano-rural.

Por outro lado, inúmeros fatores podem contribuir para amenizar essa condição, como: políticas públicas para o combate ao comércio ilícito de cigarros e o aumento dos impostos (diminui o acesso a cigarros baratos), informações aos consumidores em contrapropaganda de massa, programas de educação e controle do tabagismo nas escolas, disponibilizar terapias de reposição de nicotina e outras intervenções para a cessação tabágica etc. 22 . 
Tabela 2

Modelos de regressão de Poisson bruto e ajustado para situação censitária e prevalência de tabagismo. Brasil, 2008.

\begin{tabular}{|c|c|c|c|c|c|c|c|c|}
\hline \multirow[t]{2}{*}{ Variável } & \multicolumn{4}{|c|}{ Modelo bruto } & \multicolumn{4}{|c|}{ Modelo ajustado } \\
\hline & $\mathbf{R P}$ & IC95\% & Valor de $\mathrm{p}$ & $\begin{array}{c}\text { Valor } \\
\text { de p de } \\
\text { tendência * }\end{array}$ & RP & IC95\% & Valor de $p$ & $\begin{array}{c}\text { Valor } \\
\text { de p de } \\
\text { tendência * }\end{array}$ \\
\hline \multicolumn{9}{|l|}{ Situação censitária } \\
\hline Eminentemente rural & 1,00 & & & 0,029 & 1,00 & & & 0,042 \\
\hline Rural com influência urbana & 1,06 & $0,88-1,28$ & 0,146 & & 0,98 & $0,84-1,16$ & 0,135 & \\
\hline Urbana periférica & 0,94 & $0,88-1,04$ & 0,089 & & 0,91 & $0,76-1,09$ & 0,074 & \\
\hline Eminentemente urbana & 0,93 & $0,88-0,99$ & 0,037 & & 0,74 & $0,70-0,79$ & 0,001 & \\
\hline \multicolumn{9}{|l|}{ Sexo } \\
\hline Masculino & 1,00 & & & - & 1,00 & & & \\
\hline Feminino & 0,62 & $0,59-0,65$ & $<0,001$ & & 0,65 & $0,61-0,68$ & $<0,001$ & \\
\hline \multicolumn{9}{|l|}{ Faixa etária (anos) } \\
\hline$\leq 24$ & 1,00 & & & 0,038 & 1,00 & & & 0,043 \\
\hline $25-39$ & 1,53 & $1,41-1,65$ & $<0,001$ & & 1,56 & $1,45-1,71$ & $<0,001$ & \\
\hline $40-59$ & 2,09 & $1,94-2,26$ & $<0,001$ & & 1,96 & $1,83-2,10$ & $<0,001$ & \\
\hline$\geq 60$ & 1,32 & $1,21-1,45$ & $<0,001$ & & 1,14 & $1,04-1,26$ & $<0,001$ & \\
\hline \multicolumn{9}{|l|}{ Raça } \\
\hline Não brancos & 1,00 & & & - & 1,00 & & & \\
\hline Brancos & 0,82 & $0,79-0,86$ & $<0,001$ & & 0,92 & $0,88-0,95$ & $<0,001$ & \\
\hline \multicolumn{9}{|l|}{ Escolaridade } \\
\hline $\begin{array}{l}\leq \text { Ensino Fundamental } \\
\text { incompleto }\end{array}$ & 1,00 & & & & 1,00 & & & \\
\hline $\begin{array}{l}\geq \text { Ensino Fundamental } \\
\text { completo-Ensino Médio } \\
\text { incompleto }\end{array}$ & 0,69 & $0,65-0,74$ & $<0,001$ & $<0,001$ & 0,77 & $0,69-0,80$ & $<0,001$ & $<0,001$ \\
\hline $\begin{array}{l}\geq \text { Ensino Médio completo- } \\
\text { Ensino Superior completo }\end{array}$ & 0,51 & $0,48-0,54$ & $<0,001$ & & 0,54 & $0,48-0,59$ & $<0,001$ & \\
\hline \multicolumn{9}{|l|}{$\begin{array}{l}\text { Uso de serviços de saúde nos } \\
\text { últimos } 12 \text { meses }\end{array}$} \\
\hline Sim & 1,00 & & $<0,001$ & - & 1,00 & & $<0,001$ & - \\
\hline Não & 1,14 & $1,06-1,22$ & & & 1,12 & $1,07-1,16$ & & \\
\hline \multicolumn{9}{|l|}{ Doença relacionada ao tabaco } \\
\hline Sim & 1,00 & & 0,003 & - & 1,00 & & 0,009 & - \\
\hline Não & 1,13 & $1,04-1,19$ & & & 1,06 & $1,01-1,12$ & & \\
\hline
\end{tabular}

IC95\%: intervalo de 95\% de confiança; RP: razão de prevalência.

* Calculado para as variáveis ordinais com mais de duas categorias.

O ambiente rural pode facilitar o processo de expansão tabágica quando esta indústria patrocina eventos esportivos ou culturais na região, e a introdução do vício de forma precoce em populações que habitam próximo a áreas de cultivo 23 . Contudo, outros riscos são inerentes ao ambiente urbano como a indução e atração proporcionada pela "cultura tabágica" como um comportamento moderno, de independência e de liberdade, especialmente para jovens e mulheres; facilidade na compra do produto, inclusive os contrabandeados; e exposição ao tabagismo passivo 24.

A decisão por parar de fumar depende de vários fatores individuais e forte empenho político do poder público. Em particular, merecem destaque as medidas de controle adotadas no Brasil, que têm representado um exemplo de experiência bem-sucedida no controle do tabagismo em todo o mundo 25. Dentre essas medidas incluímos algumas de cunho educativo e/ou preventivo, outras de cunho regulador. São exemplos de medidas educativas e preventivas: inclusão de advertências sobre os perigos do consumo de tabaco nos maços de cigarros, bem como a proibição da propaganda, do 
Tabela 3

Modelos de regressão de Poisson bruto e ajustado para situação censitária e cessação de tabagismo. Brasil, 2008.

\begin{tabular}{|c|c|c|c|c|c|c|c|c|}
\hline \multirow[t]{2}{*}{ Variáveis } & \multicolumn{4}{|c|}{ Modelo bruto } & \multicolumn{4}{|c|}{ Modelo ajustado } \\
\hline & RP & IC95\% & Valor de $p$ & $\begin{array}{c}\text { Valor } \\
\text { de p de } \\
\text { tendência * }\end{array}$ & RP & IC95\% & Valor de $p$ & $\begin{array}{c}\text { Valor } \\
\text { de p de } \\
\text { tendência * }\end{array}$ \\
\hline \multicolumn{9}{|l|}{ Situação censitária } \\
\hline Eminentemente rural & 1,00 & & & 0,009 & 1,00 & & & 0,021 \\
\hline Rural com influência urbana & 1,10 & $1,03-1,18$ & 0,014 & & 1,13 & $1,06-1,20$ & 0,013 & \\
\hline Urbana periférica & 1,18 & $1,02-1,37$ & 0,009 & & 1,21 & $1,04-1,40$ & 0,006 & \\
\hline Eminentemente urbana & 1,23 & $1,05-1,45$ & 0,008 & & 1,23 & $1,07-1,39$ & $<0,001$ & \\
\hline \multicolumn{9}{|l|}{ Sexo } \\
\hline Masculino & 1,00 & & & - & 1,00 & & & - \\
\hline Feminino & 1,07 & $1,02-1,12$ & 0,003 & & 1,04 & $1,02-1,05$ & 0,009 & \\
\hline \multicolumn{9}{|l|}{ Faixa etária (anos) } \\
\hline$\leq 24$ & 1,00 & & & $<0,001$ & 1,00 & & & $<0,001$ \\
\hline $25-39$ & 1,19 & $1,08-1,33$ & $<0,001$ & & 1,18 & $1,08-1,33$ & $<0,001$ & \\
\hline $40-59$ & 1,54 & $1,39-1,70$ & $<0,001$ & & 1,52 & $1,39-1,66$ & $<0,001$ & \\
\hline$\geq 60$ & 2,05 & $1,86-2,28$ & $<0,001$ & & 2,10 & $1,92-2,29$ & $<0,001$ & \\
\hline \multicolumn{9}{|l|}{ Raça } \\
\hline Não brancos & 1,00 & & & - & 1,00 & & & - \\
\hline Brancos & 1,10 & $1,04-1,15$ & $<0,001$ & & 1,03 & $1,01-1,06$ & 0,021 & \\
\hline \multicolumn{9}{|l|}{ Escolaridade } \\
\hline $\begin{array}{l}\leq \text { Ensino Fundamental } \\
\text { incompleto }\end{array}$ & 1,00 & & & 0,008 & 1,00 & & & 0,001 \\
\hline $\begin{array}{l}\geq \text { Ensino Fundamental } \\
\text { completo-Ensino Médio } \\
\text { incompleto }\end{array}$ & 1,06 & $1,01-1,12$ & 0,020 & & 1,05 & $1,01-1,09$ & 0,032 & \\
\hline 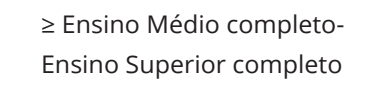 & 1,09 & 1,01 & 0,001 & & 1,12 & $1,08-1,17$ & 0,012 & \\
\hline \multicolumn{9}{|l|}{ Uso de serviços de saúde nos } \\
\hline Sim & 1,35 & $1,29-1,41$ & $<0,001$ & - & 1,29 & $1,26-1,33$ & $<0,001$ & - \\
\hline Não & 1,00 & & & & 1,00 & & & \\
\hline \multicolumn{9}{|l|}{ Doença relacionada ao tabaco } \\
\hline Sim & 1,13 & $1,07-1,18$ & $<0,001$ & - & 1,08 & $1,03-1,14$ & 0,001 & - \\
\hline Não & 1,00 & & & & 1,00 & & & \\
\hline
\end{tabular}

IC95\%: intervalo de 95\% de confiança; RP: razão de prevalência.

* Calculado para as variáveis ordinais com mais de duas categorias.

patrocínio e da promoção do tabaco. Por outro lado, como medidas regulatórias podemos citar o aumento de impostos e preços sobre os produtos do tabaco, assim como a criação de ambientes livres de tabaco, com a proibição do fumo em locais fechados 26 . É importante ressaltar que tais medidas têm um extenso marco legal, impulsionado pela ratificação feita pelo Brasil para adesão à ConvençãoQuadro para o Controle do Tabaco da Organização Mundial da Saúde (CQCT-OMS) 27. Desde esse marco, estabeleceu-se a Política Nacional de Controle do Tabaco (PNCT) e todas as leis posteriores à sua criação. Além disso, é importante considerar o Plano de Ações Estratégicas para o Enfrentamento das Doenças Crônicas Não Transmissíveis no Brasil 2011-2022 e, como consequência imediata, a criação de estratégias para que suas metas fossem cumpridas, dentre elas assegurar o tratamento para pacientes dependentes do tabaco ${ }^{28}$. Essa estratégia é assegurada no âmbito do SUS e, portanto, de acesso universal para toda a população.

Finalmente, a respeito das medidas de saúde pública existentes, é importante observar que há uma prevalência menor de doenças relacionadas ao tabaco entre quem fuma atualmente e relação 
inversa entre quem parou de fumar. Adicionalmente, há uma associação positiva entre ter acesso ao serviço de saúde nos últimos 12 meses e ter parado de fumar. Isso faz supor que as pessoas diagnosticadas com doenças relacionadas ao tabaco procuraram por serviços de saúde, primeiramente, por alguma queixa relacionada (o que levou ao diagnóstico) e, possivelmente, obteve acesso a medidas terapêuticas (farmacológicas ou não) para a cessação. Essa assunção vai ao encontro do apontado na literatura 29,30,31,32.

O trabalho tem limitações como o processo de estratificação espacial, que ocorre de praxe em categorizações de variáveis, e pode ter gerado uma perda no poder de estimação. Entretanto, é importante notar que os resultados são consistentes mesmo após o ajuste realizado com a modelagem. Há uma clara associação independente, portanto, entre a situação censitária e a prevalência e a cessação do tabagismo. Ainda, é importante destacar que há um gradiente nas medidas de prevalência e cessação entre os estratos, minimizando a possibilidade de viés na forma de classificação. Porém, há pontos de destaque como o fato desta investigação enfatizar as diferenças urbano-rurais mais complexas ao invés de se limitar a usar medidas binárias urbano-rurais 23 . Nesse sentido, essa pode ser considerada uma vantagem do presente trabalho em comparação com a maioria encontrada na literatura. Além disso, com algumas exceções como a do estudo de Sood et al. 33, dados para análise são frequentemente vistos via questionários ou pequenos subconjuntos de dados administrativos e cobertura de áreas específicas em vez de países inteiros. Ainda que a PNAD não tenha representatividade para municípios, sua abrangência é nacional.

\section{Conclusão}

Os resultados do presente estudo apontam um gradiente urbano-rural na prevalência de consumo e de cessação de tabagismo. Esse achado é importante para instrumentalizar a gestão de iniciativas de prevenção e de promoção da interrupção do comportamento, como disponibilidade de equipes de apoio, serviços de aconselhamento, disponibilidade de medicamentos e terapias auxiliares. $\mathrm{O}$ monitoramento da cessação do tabagismo é fundamental para a avaliação das ações de controle em uma população. Portanto, os resultados apontam para a necessidade de aperfeiçoar a efetividade dos serviços de acompanhamento e estímulo à cessação, especialmente para o ambiente rural.

\section{Colaboradores}

C. D. Muzi, V. C. Figueiredo e R. R. Luiz participaram da concepção do estudo, análise de dados, redação e revisão final do manuscrito.

\section{Referências}

1. World Health Organization. WHO report on the global tobacco epidemic, 2013. Enforcing bans on tobacco advertising, promotion and sponsorship http://apps.who.int/iris/bitstre am/10665/85380/1/9789241505871_eng.pdf (acessado em 04/Ago/2017).

2. Monteiro CA, Cavalcante TM, Moura EC, Claro RM, Szwarcwald CL. Population-based evidence of a strong decline in the prevalence of smokers in Brazil (1989-2003). Bull World Health Org 2007; 85:527-34. 
3. Malta DC, Vieira ML, Szwarcwald CL, Caixeta $\mathrm{R}$, Brito SMF, Reis AAC. Tendência de fumantes na população brasileira segundo a Pesquisa Nacional de Amostra de Domicílios 2008 e a Pesquisa Nacional de Saúde 2013. Rev Bras Epidemiol 2015; 18 Suppl 2:45-56.

4. Almeida L, Szklo A, Sampaio M, Souza M, Martins LF, Szklo M, et al. Global Adult Tobacco Survey data as a tool to monitor the WHO Framework Convention on Tobacco Control (WHO FCTC) implementation: the Brazilian case. Int J Environ Res Public Health 2012; 9:2520-36.

5. Levy D, de Almeida LM, Szklo A. The Brazil SimSmoke policy simulation model: the effect of strong tobacco control policies on smoking prevalence and smoking-attributable deaths in a middle income nation. PLoS Med 2012; 9:e1001336.

6. Roberts ME, Doogan NJ, Kurti AN, Redner R, Gaalema DE, Stanton CA, et al. Rural tobacco use across the United States: how rural and urban areas differ, broken down by census regions and divisions. Health Place 2016; 39:153-9.

7. Szklo AS, Levy D, Souza MC, Szklo M, Figueiredo VC, Perez C, et al. Changes in cigarette consumption patterns among Brazilian smokers between 1989 and 2008. Cad Saúde Pública 2012; 28:2211-5.

8. Szklo AS, de Almeida LM, Figueiredo VC, Autran M, Malta D, Caixeta R, et al. A snapshot of the striking decrease in cigarette smoking prevalence in Brazil between 1989 and 2008. Prev Med 2012; 54:162-7.

9. Griffin E, Moon G, Barnet R. Examining the significance of urban-rural context in tobacco quitline use: does rurality matter? Int J Public Health 2015; 60:327-33.

10. Instituto Brasileiro de Geografia e Estatística. Pesquisa Nacional por Amostra de Domicílios 2008 (PNAD 2008). http://www.ibge.gov.br/ home/estatistica/populacao/acessoainternet 2008/questionario2008.pdf (acessado em 15/ Nov/2015).
11. World Health Organization. Tobacco Free Initiative (TFI). http://www.who.int/tobacco/en/ (acessado em 20/Mar/2017).

12. Instituto Brasileiro de Geografia e Estatística. Metodologia do Censo Demográfico 2000. Série relatórios metodológicos. v. 25. http:// biblioteca.ibge.gov.br/visualizacao/livros/ liv5295.pdf (acessado em 14/Jan/2017).

13. Figueiredo VC. Um panorama do tabagismo em 16 capitais brasileiras e Distrito Federal: Tendências e heterogeneidades [Tese de Doutorado]. Rio de Janeiro: Instituto de Medicina Social, Universidade do Estado do Rio de Janeiro; 2007.

14. Idris BI, Giskes K, Borrell C, Benach J, Costa G, Federico B, et al. Higher smoking prevalence in urban compared to non-urban areas: time trends in six European countries. Health Place 2007; 13:702-12.

15. Qian J, Cai M, Gao J, Tang S, Xu L, Critchley JA. Trends in smoking and quitting in China from 1993 to 2003: National Health Servisse Survey data. Bull World Health Organ 2010; 88:769-76.

16. Pleis JR, Lucas JW. Summary health statistics for US adults: National Health Interview Survey, 2007. Vital Health Stat 10 2009; (240):1159.

17. Doescher MP, Jackson JE, Jerant A, Gary Hart L. Prevalence and trends in smoking: a national rural study. J Rural Health 2006; 22:112-8.

18. World Health Organization. Global Adult Tobacco Survey - Bangladesh. http://www. who.int/tobacco/surveillance/global_adult_ tobacco_survey_bangladesh_report_2009. pdf?ua $=1$ (acessado em 21/Mar/2017).

19. World Health Organization. Global Adult Tobacco Survey - China. http://www.who. int/tobacco/surveillance/survey/gats/en_ gats_china_report.pdf?ua=1 (acessado em 21/ Mar/2017). 
20. World Health Organization. Global Adult Tobacco Survey - India. http://www.who.int/ tobacco/surveillance/survey/gats/ind/en/ (acessado em 21/Mar/2017)

21. Tejada CAO, Ewerling F, Santos AMA, Bertoldi AD, Menezes AM. Factors associated with smoking cessation in Brazil. Cad Saúde Pública $2013 ; 29: 1555-64$.

22. Bonato AA. A fumicultura no Brasil e a Convenção-Quadro para o Controle do Tabaco. Ministério do Desenvolvimento Agrário. http://sistemas.mda.gov.br/ arquivos/1161112860.doc (acessado em 05/ Jan/2017).

23. American Legacy Fundation. Tobacco control in rural America. http://www.legacyforhealth. org/content/download/595/7129/file/ Tobacco_Control_in_Rural_America.pdf (acessado em 05/Jan/2015).

24. Horta RL, Horta BL, Pinheiro RT, Morales B, Strey MN. Tabaco, álcool e outras drogas entre adolescentes em Pelotas, Rio Grande do Sul, Brasil: uma perspectiva de gênero. Cad Saúde Pública 2007; 23:775-83.

25. Malta DC, Iser BPM, Sá NNB, Yokota RTC, Moura L, Claro RM, et al. Tendências temporais no consumo de tabaco nas capitais brasileiras, segundo dados do VIGITEL, 2006 a 2011. Cad Saúde Pública 2013; 29:812-22.

26. Malta DC, Oliveira TP, Luz MGC, Stopa SR, Silva Jr JB, Reis AAC. Tendências de indicadores de tabagismo nas capitais brasileiras, 2006 a 2013. Ciênc Saúde Coletiva 2015; 20:631-40.

27. Malta DC, Oliveira TP, Vieira ML, Almeida L, Szwarcwald CL. Uso e exposição à fumaça do tabaco no Brasil: resultados da Pesquisa Nacional de Saúde 2013. Epidemiol Serv Saúde $2015 ; 24: 239-48$.
28. Malta DC, Silva Júnior JB. O plano de ações estratégicas para o enfrentamento das doenças crônicas não transmissíveis no Brasil e a definição das metas globais para o enfrentamento dessas doenças até 2025: uma revisão. Epidemiol Serv Saúde 2013; 22:151-64.

29. Burns DM. Tobacco-related diseases. Semin Oncol Nurs 2003; 19:244-9.

30. Schiller JS, Ni H. Cigarette smoking and smoking cessation among persons with chronic obstructive pulmonary disease. Am J Health Promot 2006; 20:319-23.

31. Melzer AC, Clothier B, Japuntich SJ, Noorbaloochi S, Hammett P, Burgess DJ, et al. Comparative effectiveness of proactive tobacco treatment among smokers with and without chronic lower respiratory disease. Ann Am Thorac Soc 2018; 15:341-7.

32. Phillips E, Wang TW, Husten CG, Corey CG, Apelberg BJ, Jamal A, et al. Tobacco product use among adults - United States, 2015. MMWR Morb Mortal Wkly Rep 2017; 66:1209-15.

33. Sood A, Andoh J, Rajoli N, Hopkins-Price P, Verhulst SJ. Characteristics of smokers calling a national reactive telephone helpline. Am J Health Promot 2008; 22:176-9. 
Abstract

The degree of urbanization has been associated with both tobacco consumption and quit rates. The current study aims to examine the importance of the urban-rural context in the determination of tobacco consumption patterns and smoking cessation. Data were used from the supplement of the Special Survey on Smoking (PETab) from the 2008 Brazilian National Household Sample Survey (PNAD). The sample included 14,420 individuals over 15 years of age, of whom 7,003 were current smokers and 7,417 former smokers in the PETab. Smoking prevalence and cessation rates were calculated. Individuals were stratified according to urban and rural residence. Other sociodemographic variables were also analyzed: sex, age bracket, race, and schooling. To explore the difference in smoking prevalence and cessation rate for each stratum in the census situation, the data were adjusted using a Poisson model. Smoking prevalence was higher in places less influenced by the urban environment. Meanwhile, smoking cessation was higher in more urbanized places. Smoking prevalence showed a decrease in consumption as the population's urban characteristics increased $(P R=0.75$ for eminently urban vs eminently rural, $p=0.001$ ). Smoking cessation trends showed that the more urban population was more prone to quitting $(P R=1.22$ for eminently urban vs eminently rural, $p<0.001)$. The results point to the need to improve the effectiveness of services for follow-up and encouragement of cessation in rural areas.

Smoking; Smoking Cessation; Tobacco Use Disorder; Urban Population; Rural Population

\section{Resumen}

El grado de urbanización ha estado asociado al consumo de productos derivados del tabaco, así como a la interrupción del hábito de fumar. Así pues, el objetivo de este trabajo ha sido examinar la importancia del contexto urbano-rural para determinar el patrón de consumo de derivados del tabaco, así como el fin del hábito de fumar. Se utilizaron datos del módulo complementario de la Encuesta Especial sobre Tabaquismo (PETab por sus siglas en portugués) de la Encuesta Nacional por Muestras de Domicilios (PNAD) de 2008. Se analizaron a 14.420 individuos mayores de 15 años, siendo 7.003 tabaquistas actuales y 7.417 extabaquistas en la PETab. Se calculó la prevalencia y el indice en el cese de tabaquismo. Hubo una estratificación espacial de esos individuos como residentes en ambientes urbanos y rurales. Se analizaron también otras variables sociodemográficas: sexo, franja de edad, raza y escolaridad. Para explorar las diferencias entre la prevalencia, y el fin del tabaquismo en cada estrato dentro de su situación censal, los datos se ajustaron usando el modelo de Poisson. Existe una prevalencia de tabaquismo mayor en los lugares menos influenciados por el ambiente urbano. Por otro lado, la eliminación del tabaquismo es mayor en los lugares más urbanizados. Se observa que para la prevalencia del tabaquismo existe una reducción en el consumo, a medida que aumenta la característica urbana de la población $(R P=0,75$ para eminentemente urbana vs. eminentemente rural, $p=0,001)$. Para el fin del tabaquismo, se observa que la población más urbana es la más propensa a la interrupción del consumo $(R P=1,22$ para eminentemente urbana vs. eminentemente rural, $p<0,001)$. Los resultados apuntan la necesidad de perfeccionar la efectividad de los servicios de acompañamiento y estímulo al fin del tabaquismo en el ambiente rural.

Fumar; Cese del Hábito de Fumar; Tabaquismo; Población Urbana; Población Rural
Recebido em 08/Mai/2017

Versão final reapresentada em 28/Nov/2017

Aprovado em 26/Dez/2017 